\title{
NECESSIDADE DE TRATAMENTO ODONTOLÓGICO E NÍVEIS DE ANSIEDADE EM ADOLESCENTES
}

\author{
DENTAL TREATMENT NEEDS AND ANXIETY \\ IN BRAZILIAN ADOLESCENTS
}

\section{Raphael Lobo Lobo de Souza', Ana Rita Duarte Guimarães², Paulo Carvalho Tobias Duarte ${ }^{3}$, Magali Teresópolis Reis Amaral ${ }^{4}$, Hervania Santana da Costa ${ }^{5}$, Raylene Laíse Souza Silva ${ }^{6}$}

Autor para correspondência: Raphael Lôbo de Souza - rapha_lobo_2011@hotmail.com 'Graduando em Odontologia pela Universidade Estadual de Feira de Santana. Feira de Santana, Bahia, Brasil. ${ }^{2}$ Doutora em Odontologia. Professora na Universidade Estadual de Feira de Santana. Feira de Santana, Bahia, Brasil. ${ }^{3}$ Doutor em Ciência Odontológica. Professor na Universidade Estadual de Feira de Santana. Feira de Santana, Bahia, Brasil. ${ }^{4}$ Mestre em Biometria e Estatística Aplicada. Professora na Universidade Estadual de Feira de Santana. Feira de Santana, Bahia, Brasil. ${ }^{5}$ Doutora em Saúde Coletiva. Professora na Universidade Estadual de Feira de Santana. Feira de Santana, Bahia, Brasil. ${ }^{6}$ Cirurgiã-dentista. Feira de Santana, Bahia, Brasil.

\begin{abstract}
Objective: to evaluate the relationship between the need for dental treatment due to dental caries and anxiety levels in adolescents. Method: this cross-sectional study examined 89 adolescents aged 11 to 19 years. Those responsible for the adolescent signed an informed consent form for the inclusion of adolescent in the study. Dental anxiety was estimated using the Corah Dental Anxiety Scale (DAS). Data were collected and analyzed by SPSS, using descriptive statistics and Pearson's chi-square test. Results: 1 adolescent (1.1\%) did not present anxiety (DAS $=0$ ); 40 adolescents $(44.9 \%)$ had low levels (DAS $=5-9), 39(43.8 \%)$ moderate levels (DAS $=10-15)$ and $9(10.1 \%)$ high levels (DAS $=16-20)$. The girls presented higher levels of anxiety than the boys $(p=0.04)$. The mean CPO-d was 2.69. 39 adolescents $(43.8 \%)$ presented dental treatment needs. The relationship between the need for dental treatment and anxiety was statistically significant $(p=0.003)$. More adolescents with treatment needs were observed in moderate and high anxiety levels (28.1\%). Additionally, the need for dental treatment due to caries was significantly higher among adolescents with higher levels of dental anxiety than those with lower anxiety levels $(p=0.001)$. Conclusion: there was a statistically significant relationship between the need for dental treatment and anxiety. Female adolescents had higher levels of anxiety.
\end{abstract}

Keywords: Anxiety; Dental Caries; Adolescent 


\section{INTRODUÇÃO}

A ansiedade é a reação básica do ser humano frente a um perigo ou ameaça, podendo assumir formas e intensidades variadas'. A experiência ao tratamento odontológico tem sido relatada por muitos pacientes como uma condição geradora de estresse e de ansiedade ${ }^{2}$. Adicionalmente, a ansiedade tem sido considerada um fator preponderante para $\circ$ cancelamento de consultas odontológicas por adolescentes americanos entre 12 e 18 anos de idade ${ }^{3}$. Estudos têm demonstrado que hábitos pouco saudáveis na adolescência se constituem em fator de risco para o desenvolvimento de doenças na vida adulta. Neste período, medidas adequadas de higiene bucal podem entrar em conflito com o estilo de vida dos adolescentes não tão receptivos à supervisão dos responsáveis ${ }^{4}$.

Etnia, baixa renda familiar, baixa escolaridade dos pais, experiência de cárie e medo ao tratamento odontológico têm sido associados ao relato de dor de dente ${ }^{5}$. A dor na cavidade bucal parece ser 0 problema de saúde bucal de maior impacto sobre o bem-estar dos indivíduos, interferindo diretamente na qualidade de vida, pois provoca desordens no sono, diminuição do rendimento no trabalho, faltas escolares e dificuldades na alimentação ${ }^{5}$. Além disso, a falta de interação paciente-profissional e a sensação de ter parte do corpo invadida pelo dentista foram considerados fatores desencadeadores de ansiedade para adolescentes ${ }^{5}$.

O papel do cirurgião-dentista não deveria se limitar à execução do tratamento bucal, mas incluir a identificação de situações geradoras de ansiedade para $\circ$ paciente e suas possíveis origens ${ }^{7}$. Observase que um profissional preocupado apenas com 0 procedimento a ser realizado pode não perceber manifestações de ansiedade e, por isso, não oferecer - amparo necessário e imediato ao paciente ${ }^{8}$. De modo geral, é aceito que o medo da dor, a partir de uma experiência desconfortável no passado, é o fator principal da causa da ansiedade odontológica e é responsável pela maioria dos casos de pacientes que evitam o tratamento odontológico?.

Este trabalho teve como objetivo avaliar a relação entre a necessidade de tratamento odontológico devido à cárie dental e níveis de ansiedade em adolescentes.

\section{MATERIAL E MÉTODO}

Tratou-se de um estudo de corte transversal (fluxograma apresentado na Figura 1) realizado com adolescentes do Colégio Estadual José Ferreira Pinto, localizado na cidade de Feira de Santana (BA). Seguindo os critérios de inclusão e exclusão (Figura 1), adolescentes na faixa etária de 11 a 19 anos foram selecionados. A coleta de dados foi realizada na própria escola e no ambulatório da Clínica Integrada do Curso de Odontologia da Universidade Estadual de Feira de Santana no período de março a junho de 2014 . Foi aplicado um formulário socioeconômico (ABIPEME) e um formulário baseado na escala de ansiedade dental de Corah, Dental Anxiety Scale (DAS), escala psicométrica que consiste de quatro perguntas de múltiplaescolha relacionadas com as reações subjetivas do paciente diante da consulta odontológica. Cinco respostas com valor de um a cinco foram fornecidas, categorizando de forma diretamente proporcional à ansiedade 10. Foram atribuídos escores de 0 a 5 para cada alternativa de cada questão (com o melhor resultado equivalente a 4 e o pior a 20). Os seguintes critérios foram considerados para se determinar 0 grau de ansiedade dental: até 4 pontos ausência de ansiedade, de 5 a 9 baixa ansiedade, de 10 a 15 ansiedade moderada e de 16 a 20 alta ansiedade dental.

Após a aplicação dos formulários, procedeu-se 0 exame clínico, para conhecimento do índice CPO-d e necessidade de tratamento dos adolescentes pesquisados, seguido do preenchimento da ficha de exame preconizada pelo Ministério da Saúde ${ }^{11}$. O exame bucal foi feito sob luz artificial com auxílio de espelho, pinça clínica e sonda OMS.

A seguir, foi realizada a análise dos dados, para verificar a relação entre o percentual de cárie/ necessidade de tratamento e os níveis de ansiedade 
registrados pelo índice DAS. Para calcular as diferenças de proporções, aplicou-se o teste qui-quadrado de Pearson com nível de significância de $5 \%$. As informações foram sistematizadas em tabelas e gráficos com o auxílio do SPSS (Statistical Package for the Social Sciences) e do Programa Microsoft Excel 2007, seguindo a distribuição das variáveis de estudo, para apresentação das informações obtidas.

Para os adolescentes e responsáveis foi entregue o termo de consentimento livre e esclarecido de acordo com a resolução 196/96 do Conselho Nacional de Saúde. Este estudo foi aprovado pelo comitê de ética da Universidade Estadual de Feira de Santana (108/2009; CAAE 011 15.0.059.00009).

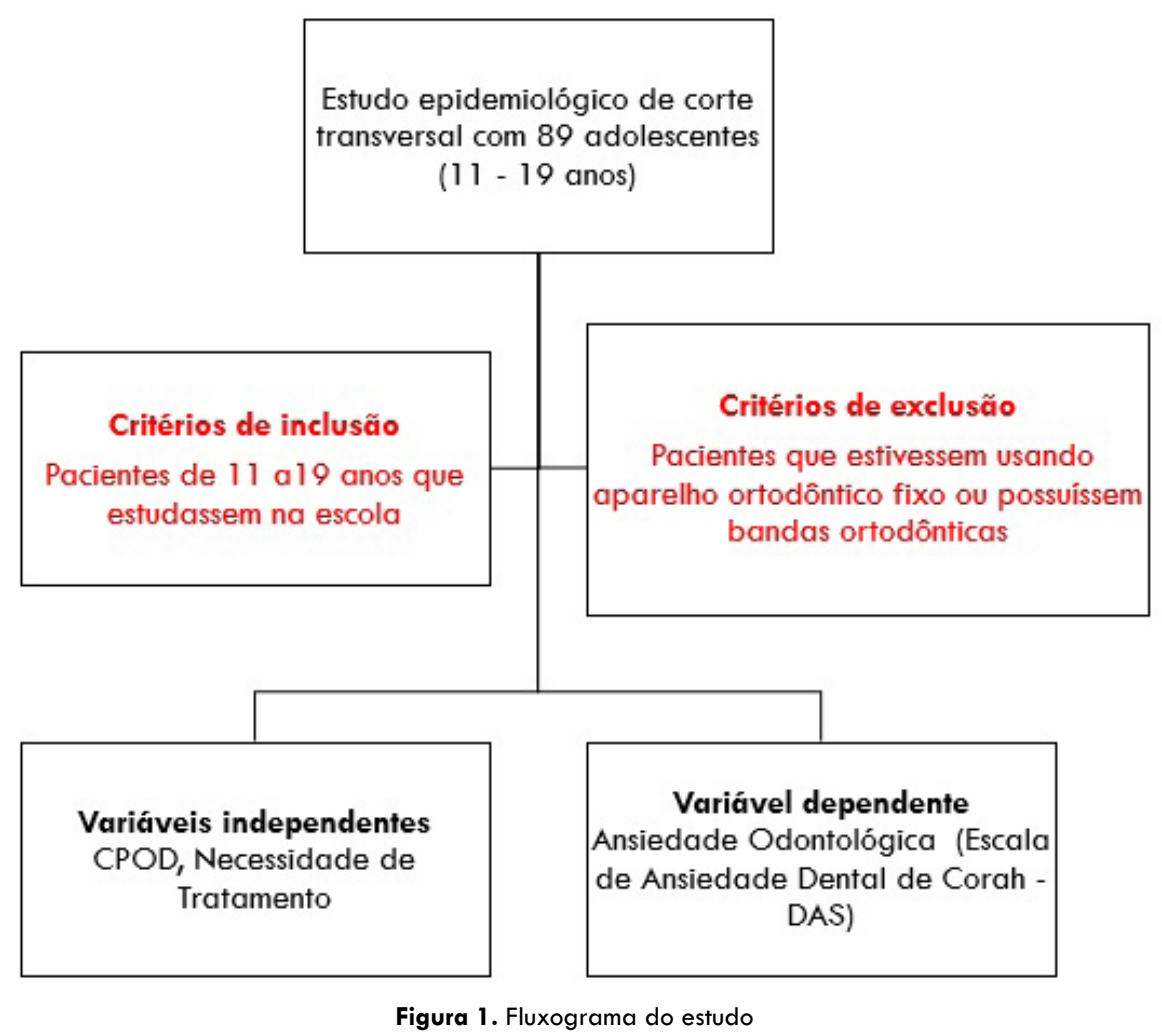

\section{RESULTADO}

O grupo de estudo foi composto por 89 adolescentes de 11 a 19 anos de idade, sendo 51 (57,3\%) do sexo feminino e $38(42,7 \%)$ do sexo masculino. Observouse que grande parte dos adolescentes estava inserida em famílias de classes baixa e média, uma vez que $51 \quad(57,3 \%)$ dos entrevistados relataram ter renda mensal entre 0 e 3 salários mínimos. Quanto ao escore de ansiedade, observou-se que 1 adolescente $(1,1 \%)$ não apresentou ansiedade (DAS $=0), 40$ adolescentes $(44,9 \%)$ tiveram níveis baixos (DAS $=5-9$ ), $39(43,8 \%)$ níveis moderados (DAS $=10-15)$ e $9(10,1 \%)$ níveis elevados (DAS = 16-20) (Tabela 1). O CPO-D médio foi de 2,69.
Do total, 39 adolescentes $(43,8 \%)$ apresentaram necessidade de tratamento odontológico.

Na análise das características sociodemográficas, observou-se uma associação estatisticamente significante entre sexo e DAS $(p=0,038)$ e entre classe socioeconômica e grau de ansiedade dental $(p=0,012)$ (Tabela 1).

Quanto ao histórico odontológico e biopsicossocial, verificou-se que a relação entre CPO-d e DAS (escala de ansiedade dental de Corah) não foi estatisticamente significante $(p=0,631)$, bem como 
a relação entre sexo e CPO-d $(p=0,292)$ (Tabelas 2 e 3).

Nesta pesquisa não houve associação estatisticamente significante entre CPO-d e ansiedade dental, porém quando se analisou os componentes $C$ (dente cariado) e $P$ (dente perdido) separadamente, identificou-se um valor de $p$ estatisticamente significante para o componente $C(p=0,001)$ e para o componente $P(p=0,002)$; (Tabela 2).

Tabela 1. Características do grupo de estudo, total e por nível de ansiedade odontológica, Feira de Santana, Bahia, $2015(n=89)$

\begin{tabular}{|c|c|c|c|c|c|c|c|c|c|c|c|}
\hline \multirow[b]{3}{*}{ Características } & & & \multicolumn{8}{|c|}{ Ansiedade Odontológica } & \multirow{3}{*}{$\begin{array}{l}\text { Valor } \\
\text { de } p^{\circ}\end{array}$} \\
\hline & \multicolumn{2}{|c|}{ Total } & \multicolumn{2}{|c|}{ Alto } & \multicolumn{2}{|c|}{ Baixo } & \multicolumn{2}{|c|}{ Moderado } & \multicolumn{2}{|c|}{ Nulo } & \\
\hline & $\mathbf{n}$ & $\%$ & $\mathrm{n}$ & $\%$ & $\mathbf{n}$ & $\%$ & $\mathbf{n}$ & $\%$ & $\mathrm{n}$ & $\%$ & \\
\hline \multicolumn{12}{|l|}{ Sexo } \\
\hline Feminino & 51 & 57,30 & 9 & 10,10 & 21 & 23,60 & 20 & 22,50 & 1 & 1,10 & $0,038^{b}$ \\
\hline Masculino & 38 & 42,70 & 0 & 0,00 & 19 & 21,30 & 19 & 21,30 & 0 & 0,00 & \\
\hline \multicolumn{12}{|l|}{ Classe } \\
\hline \multicolumn{12}{|l|}{ Socioeconômica } \\
\hline Menos de 1 salário & 23 & 25,80 & 3 & 3,40 & 12 & 13,50 & 7 & 7,90 & 1 & 1,10 & $0,012^{b}$ \\
\hline De 1 a 3 salários & 51 & 57,30 & 5 & 5,60 & 16 & 18,00 & 30 & 33,70 & 0 & 0,00 & \\
\hline Mais de 3 salários & 15 & 16,90 & 1 & 1,10 & 12 & 13,50 & 2 & 2,20 & 0 & 0,00 & \\
\hline
\end{tabular}

${ }^{a}$ Teste qui-quadrado $(x 2)$ de Pearson para diferenças de proporções; ${ }^{b} p<0,05$ (estatisticamente significante)

Tabela 2. Necessidade de tratamento e CPO-d do grupo de estudo, total e por nível de ansiedade odontológica, Feira de Santana, Bahia, 2015 ( $n=89$ ).

\begin{tabular}{|c|c|c|c|c|c|c|c|c|c|c|c|}
\hline \multirow[b]{3}{*}{ Características } & & & \multicolumn{8}{|c|}{ Ansiedade Odontológica } & \multirow{3}{*}{$\begin{array}{l}\text { Valor } \\
\text { de } p^{a}\end{array}$} \\
\hline & \multicolumn{2}{|c|}{ Total } & \multicolumn{2}{|r|}{ Alto } & \multicolumn{2}{|c|}{ Baixo } & \multicolumn{2}{|c|}{ Moderado } & \multicolumn{2}{|c|}{ Nulo } & \\
\hline & n & $\%$ & $\mathbf{n}$ & $\%$ & $\mathbf{n}$ & $\%$ & $\mathbf{n}$ & $\%$ & $\mathbf{n}$ & $\%$ & \\
\hline \multicolumn{12}{|l|}{ Necessidade de } \\
\hline \multicolumn{12}{|l|}{ Treatamento } \\
\hline Sim & 39 & 43,80 & 9 & 10,10 & 14 & 15,70 & 16 & 18,00 & 0 & 0,00 & $0,003^{b}$ \\
\hline Não & 50 & 56,20 & 0 & 0,00 & 26 & 29,20 & 23 & 25,80 & 1 & 1,10 & \\
\hline \multicolumn{12}{|l|}{ CPO-d } \\
\hline Baixo & 67 & 75,30 & 5 & 5,60 & 33 & 37,10 & 28 & 31,50 & 1 & 1,10 & 0,631 \\
\hline Moderado & 12 & 13,50 & 2 & 2,20 & 3 & 3,40 & 7 & 7,90 & 0 & 0,00 & \\
\hline Alto & 10 & 11,20 & 2 & 2,20 & 4 & 4,50 & 4 & 4,50 & 0 & 0,00 & \\
\hline \multicolumn{12}{|l|}{ C do CPO-d } \\
\hline Sim & 52 & 58,40 & 9 & 10,10 & 28 & 31,50 & 23 & 25,80 & 0 & 0,00 & $0,001^{b}$ \\
\hline Não & 37 & 41,60 & 0 & 0,00 & 12 & 13,50 & 16 & 18,00 & 1 & 1,10 & \\
\hline \multicolumn{12}{|l|}{ p do CPO-d } \\
\hline Sim & 77 & 86,50 & 4 & 4,50 & 37 & 41,60 & 35 & 39,30 & 0 & 0,00 & $0,002^{b}$ \\
\hline Não & 12 & 13,50 & 5 & 5,60 & 3 & 3,40 & 4 & 4,50 & 1 & 1,10 & \\
\hline \multicolumn{12}{|l|}{ O do CPO-d } \\
\hline Sim & 34 & 38,20 & 4 & 4,50 & 17 & 19,10 & 13 & 14,60 & 0 & 0,00 & 0,689 \\
\hline Não & 55 & 61,80 & 5 & 5,60 & 23 & 25,80 & 26 & 29,20 & 1 & 1,10 & \\
\hline
\end{tabular}

${ }^{a}$ Teste qui-quadrado ( $x 2$ ) de Pearson para diferenças de proporções; ${ }^{b} p<0,05$ (estatisticamente significante) 


\begin{tabular}{|c|c|c|c|c|c|c|c|c|c|}
\hline \multirow[b]{3}{*}{ Características } & \multicolumn{8}{|c|}{ CPO-d } & \multirow[t]{3}{*}{ Valor de $\mathrm{p}^{\circ}$} \\
\hline & \multicolumn{2}{|c|}{ Baixo } & \multicolumn{2}{|c|}{ Moderado } & \multicolumn{2}{|c|}{ Alto } & \multicolumn{2}{|c|}{ Total } & \\
\hline & $\mathbf{n}$ & $\%$ & $\mathbf{n}$ & $\%$ & $\mathbf{n}$ & $\%$ & $\mathrm{n}$ & $\%$ & \\
\hline \multicolumn{10}{|l|}{ Sexo } \\
\hline Feminino & 36 & 40,40 & 7 & 7,90 & 8 & 9,00 & 51 & 57,30 & 0,292 \\
\hline Masculino & 31 & 34,80 & 5 & 5,60 & 2 & 2,20 & 38 & 42,70 & \\
\hline
\end{tabular}

${ }^{a}$ Teste qui-quadrado (x2) de Pearson para diferenças de proporções; ${ }^{b} p<0,05$ (estatisticamente significante)

\section{DISCUSSÃO}

Neste estudo foi observada relação estatisticamente significante entre necessidade de tratamento odontológico e ansiedade, indicando que adolescentes com alto índice de ansiedade dental, precisam de maior necessidade de tratamento, - que está de acordo com estudo prévio destes autores ${ }^{14}$. Isto pode estar relacionado ao fato de que adolescentes mais ansiosos podem evitar $\circ$ tratamento odontológico por medo, adquirindo menos conhecimento acerca da importância da saúde bucal.

O conhecimento da situação de saúde bucal de uma população e dos fatores que podem influenciá-la é essencial para promover mudanças de comportamento que levem a atitudes positivas em relação aos cuidados com a mesma. A garantia de acesso e a qualificação contínua dos serviços de saúde bucal são imprescindíveis na busca de melhores condições de vida e saúde para nossos jovens ${ }^{12}$.

Nesta pesquisa, observou-se que a variável sexo esteve diretamente relacionada com $\circ$ grau de ansiedade, pois os indivíduos do sexo feminino apresentaram escores mais altos do que os do sexo masculino, dado também obtido em estudo prévio ${ }^{13}$, podendo indicar que as meninas expõem mais suas emoções que os meninos. Analisando-se a relação entre sexo e CPO-d, percebeu-se que as meninas apresentavam índices mais elevados do que os meninos, o que pode estar relacionado ao maior grau de ansiedade das meninas.
Na presente pesquisa, foi constatado que pacientes que não mostraram ansiedade dental, também não apresentaram dentes perdidos, cariados ou obturados, podendo-se concluir que estes adolescentes eram mais esclarecidos quanto aos hábitos saudáveis de higiene bucal. Por outro lado, outros estudos verificaram que grupos de adolescentes com relato de dor apresentaram piores condições de saúde bucal, confirmado por suas necessidades de tratamento e maior experiência de cárie (CPO-d $)^{5,15}$.

A condição socioeconômica é um dos determinantes sociais mais importantes na utilização de serviços odontológicos. O percentual de indivíduos com ansiedade ao tratamento odontológico varia de um contexto para outro, provavelmente em decorrência das diferenças socioculturais ${ }^{8}$. Nesta pesquisa, adolescentes pertencentes à classe socioeconômica mais favorecida apresentaram o menor percentual de ansiedade da amostra que pode ser atribuído à maior utilização dos serviços odontológicos por populações com maior poder aquisitivo. Em estudo nacional sobre utilização de serviços, observou-se que o número de desassistidos era muito maior entre os mais pobres, e esses eram também os que tinham maior dificuldade de conseguir atendimento quando o buscavam ${ }^{16}$.

Este estudo foi feito com uma amostra de escolares, restrita, mas que suscitou a ideia da relação entre necessidade de tratamento e ansiedade. Fazem-se necessários estudos com uma amostra maior, pois, 
trata-se de um tema que deve ser amplamente estudado frente os conflitos psicológicos enfrentados pelos indivíduos na fase da adolescência.

\section{CONCLUSÃO}

Neste estudo, verificou-se relação estatisticamente significante entre necessidade de tratamento odontológico e ansiedade. Pacientes menos ansiosos apresentaram índice CPO-d menor do que os adolescentes com maiores escores de ansiedade dental e, consequentemente, menor necessidade de tratamento.

\section{CONTRIBUIÇÕES DOS AUTORES}

Silva RLS, Guimarães ARD, Duarte PCT e Costa HS participaram da concepção, delineamento, coleta e interpretação dos dados, assim como da confecção da redação e manuscrito do artigo científico. Amaral MTR participou da interpretação e análise dos dados. Souza RL participou da redação, confecção do manuscrito e encaminhamento do artigo científico para publicação.

\section{CONFLITOS DE INTERESSES}

Nenhum conflito financeiro, legal ou político envolvendo terceiros (governo, empresas e fundações privadas, etc.) foi declarado para nenhum aspecto do trabalho submetido (incluindo mas não limitandose a subvenções e financiamentos, conselho consultivo, desenho de estudo, preparação de manuscrito, análise estatística, etc).

\section{REFERÊNCIAS}

1. Batista MA, Sisto FF. Estudo para construção de uma escala para adolescentes. Estud Psicol. 2005; 22(4):347-54. doi: 10.1590/S0103-166X2005000400002

2. Moraes ABA, Costa Junior AL, Rolim GS. Medo de dentista: ainda existe? In: Brandão MZS. Sobre Comportamento e cognição. Santo André: Esetec. 2004; 14(3):171-8.

3. Ramos-Jorge MLR, Cardoso M, Marques LS, Bosco VL, Rocha MJC. Associação entre experiência odontológica na infância e ansiedade odontológica na adolescência. Arq Odontol. 2004;40(3): $111-206$.

4. Freddo SL, Aerts DRGC, Abbeg C, Davoglio R, Vieira PC, Monteiro L. Hábitos de higiene bucal e utilização de serviços odontológicos em escolares de uma cidade da região sul do Brasil. Cad Saúde Pública. 2008;24(9):1991-2000. doi: $10.1590 / \mathrm{S} 0102-311 \times 2008000900005$

5. Rhis LB, Cypriano S, Souza MLR, Silva RC, Gomes PR. Dor de dente e sua relação com experiência de cárie em adolescentes. RGO. 2008;56(4):361-5.

6. Klatchoian, DA. A relação dentista-paciente. In: Klatchoian DA. Psicologia Odontopediátrica. São Paulo: Santos. 2002;18(4):13-27.

7. Possobon RF, Carrascoza KC, Moraes ABA, Costa Jr AL. O tratamento odontológico como gerador de ansiedade. Psicol Estud. 2007;1 2(3):609-16.

8. Bottan ER, Oglio JD, Araújo SM. Ansiedade ao tratamento odontológico em estudantes do ensino fundamental. Pesq Bras Odontoped Clin Integr. 2007;7(3):241-6. doi: 10.4034/1519. $\underline{0501.2007 .0073 .0008}$

9. Ferreira CM, Filho EDG, Valverde GB, Moura EH, Deus G, Filho TC. Ansiedade odontológica: nível, prevalência e comportamento. RBPS. 2004; 17(2):51-5.

10. Torriani DD, Teixeira AM, Pinheiro R, Goettems ML, Bonow $M L M$. Adaptação transcultural de instrumentos para mensurar ansiedade e comportamento em clínica odontológica infantil. Arq Odontol. 2008;44(4):17-23.

11. Brasil. Ministério da Saúde. Secretaria de Políticas de Saúde. Departamento de Atenção Básica, Área Técnica de Saúde Bucal. Projeto SB2000: condições de saúde bucal da população brasileira no ano 2000: manual do examinador. Brasília. DF. 2001.

12. Davoglio RS, Aerts DRGC, Abbeg C, Freddo SL, Monteiro L. Fatores associados à hábitos de saúde e utilização de serviços odontológicos entre adolescentes. Cad Saúde Pública. 2009;25(3):655-67. doi: 10.1590/S0102311X2009000300020

13. Singh KA, Moraes ABA, Ambrosano GMB. Medo, ansiedade e controle relacionados ao tratamento odontológico. Pesq Odont Bras. 2000;1 4(2):131-6.

14. Guimarães ARD, Jorge-Ramos ML, Cardoso M, Cordeiro $M$, Vieira RS. Relação entre a necessidade de tratamento odontológico e a ansiedade em adolescentes. Pesq Bras Odontoped Clin Integr. 2009;9(supl):37-50.

15. Nomura LH, Bastos JLD, Peres MA. Dental pain prevalence and Association with dental caries and socioeconomic status in school children. Southern Brazil. Bras Oral Res. 2004;18(2):134-40. doi: 10.1590/S1806$\underline{83242004000200008}$

16. Barros AJD, Bertoldi AD. Desigualdades na utilização e no acesso a serviços odontológicos: uma avaliação em nível nacional. Ciênc Saúde Coletiva. 2002;7(4):709-17. doi: 10.1590/S1413-81232002000400008 